\title{
Conductance and supercurrent discontinuities in atomic size point contacts
}

\author{
C.J. Muller ${ }^{\mathrm{a}}$, J.M. van Ruitenbeek ${ }^{\mathrm{a}}$, C.W.J. Beenakker ${ }^{\mathrm{b}}$ and R. de Bruyn Ouboter ${ }^{\mathrm{a}}$ \\ ${ }^{3}$ Kamerlingh Onnes Laboratory, ${ }^{\mathrm{b}}$ Instituut-Lorentz, Untversity of Letden, Leiden, The Netherlands
}

\begin{abstract}
A technique is described, which uses the adjustable stress in a bending beam to control the constriction diameter betwcen two electrodes The controllability of the constriction diameter is useful when a large range in the normal resistance of different junctions is required for a systematic study In the high resistance point contact regime quantum size effects are studied in metalic and superconducting constrictions When changing the constriction diameter reproducible steps in the conductance of the order of $2 e^{2} / h$ are observed For superconducting point contacts concomitant jumps in the supercurrent of order $e \Delta / \hbar$ are seen For contacts adjusted at a step the conductance switches in time between two values, which are interpreted as "two level fluctuations" in the site of a single atom in the constriction
\end{abstract}

\section{Introduction}

The conductance of a narrow contact under the condition of ballistic motion of the electrons, changes by integer multiples of $2 e^{2} / h$ as a function of the width of the contact. This quantization of the conductance was discovered in point contacts of high mobility two-dimensional electron gases in GaAs-AlGaAs heterostructures [1], having a large Fermi wavelength $\left(\lambda_{F}=\right.$ $400 \AA$ ). Its explanation is based on the quantization of the transverse momentum of the electrons, which move through the contact. This principle is expected to apply to all wave phenomena. Indeed, also the transmission of light through a narrow aperture was subsequently discovered to be quantized [2]. Quantization of the conductance is now understood by means of the Landauer formalism [3]. The wave character of the electrons, which manifests itself so strongly in the conductance of these mesoscopic structures, should also show up in other transport properties. In particular, Beenakker and van Houten [4] predicted that the critical current of a

Correspondence to $\mathrm{C} \mathrm{J}$ Muller, Kamerlıngh Onnes Laboratory, University of Leiden, P O Box 9506, 2300 RA Leiden, The Netherlands short, impurity free superconducting constriction should increase stepwise as a function of its width. The step size was found to depend on the superconducting energy gap, $\Delta$, as $e \Delta / \hbar$.

In principle, quantization of the conductance is also expected for point contacts of normal metals. This is more difficult to observe, however, as the Fermi wavelength in metals is of the order of the atomic diameter. To have constriction widths of the order of the Fermi wavelength implies that one should construct point contacts of atomic dimensions for which the diameter can be continuously adjusted. Variation of the constriction diameter on such a fine scale will suffer from the limits set by the size of the atoms. Conduction quantization effects in metals will therefore be blurred by discrete atom-by-atom building up of the contact. Nevertheless, it is of considerable interest to study such small metallic point contacts. Some interesting questions to be investigated are: what is the number of quantum channels for conduction through one atom? How is this number related to the atomic shell structure? Can we see the effects of conductance quantization? A metal point contact also offers the possibility to look for steps in the supercurrent, a subject which is not yet accessible with semiconductor devices. 
Here, an experimental method is described which makes it possible to study atomic size point contacts of any metal, and where the contact size can be adjusted continuously. First attempts at observing conductance quantization in small normal metal and superconducting point contacts are presented. When the point contact constriction is increased the conductance is observed to increase stepwise. The steps are interpreted as atom-by-atom increase of the contact diameter. The step size for a platinum point contact is of order $2 e^{2} / h$. In a niobium superconducting point contact the steps in the normal state conductance are found to coincide with steps in the supercurrent, with a step size of the order of the predicted value [4].

In the next section the experimental technique, which is used to obtain atomic size point contacts, is described in detail. Experiments on these contacts in the normal and the superconducting state are presented in the last two sections. Parts of this paper have been published elsewhere $[5,6]$.

\section{The experimental technique}

Here we describe an extension to Morelands break junction technique [7], which is used to obtain a clean and stable adjustable junction. A crucial part in the setup is the sample mounting, which is schematically drawn in fig. 1. A metal filament of the material under investigation is glued on a flexible glass bending beam, in such a way that the central section remains unglued. Prior to this procedure a notch is made in the middle of the filament with a sharp scalpel. The principle of operation is to break the notched part of the metal filament at liquid helium temperatures, in vacuum, in order to obtain two clean freshly broken electrodes. This is established by bending the substrate in a controllable way, resulting in an elongation of the unglued section $u$. This elongation is concentrated on the weak notched part in the filament, and results in the fracture, which is measured as an abrupt decrease of the conductivity to zero. After the fracture of the electrodes the force, which acts

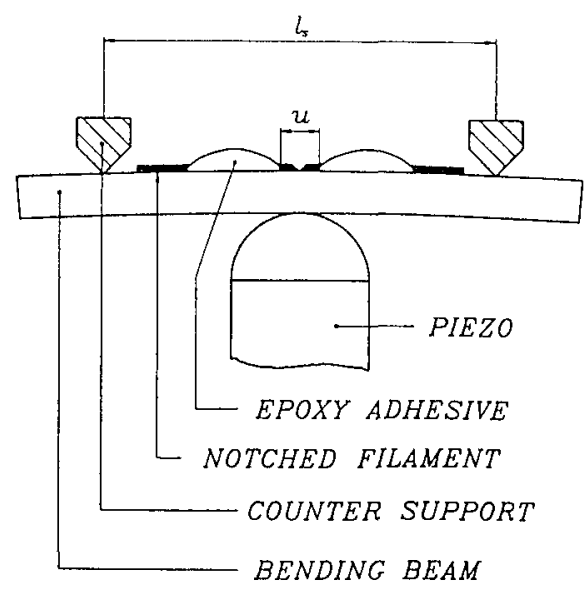

Fig 1 A schematic drawing of the sample mounting in a three point bending configuration $A$ force on the bending beam, exerted vid the piezo element, induces a small length increase of the unglued section $u$ to break the filament Once this is established the force on the bending beam is used for fine adjustment of a point contact or a tunnel junction The distance between the counter supports, $l_{\mathrm{s}}$, is approximately $2 \mathrm{~cm}$

on the bending beam, is relaxed until the two electrode halves touch again. Once this is achieved the bending beam is still under a strain. By fine adjustment of the force acting on the bending beam, via a piezo element, it is possible to adjust atomic size point contacts or tunnel junctions with a subatomic vacuum distance as the barrier.

Two possible electrode geometries are indicated in fig. 2. Fig. 2(a) represents a tunnel junction, where electron transport takes place via tunneling, in contrast to the point contact shown in fig. 2(b) where conduction is the transport mechanism. On an atomic scale the electrode surfaces are rough. Therefore, in the tunnel regime tunneling occurs somewhere between two nearest atoms located on opposite electrodes. When the vacuum distance is decreased these two atoms start to form an atomic size point contact. Due to the stability and adjustability of the system it is possible to establish the transition from a vacuum barrier to a contact, opening the possibility to study the transition from tunneling to conduction [5]. A retardation factor imposed by the sample geome- 


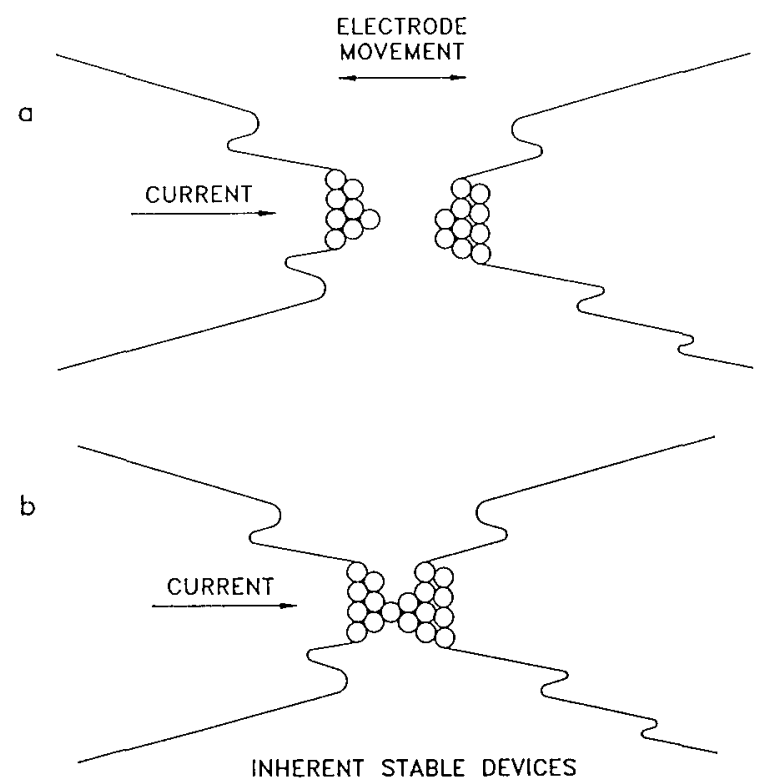

Fig 2 Two possible clectrode geometises The clectrodes are tightly connected to the bending beam and form an inherently stable structure In (a) the two electiodes constitute a tunnel function, tunneling occurring somewhere between two nearest atoms on either side of the cledn electrodes In (b) the two electrodes constitute an atomic stze point contact, where election tiamsport takes place vla conduction.

try is the reason for the high stability of the system and for the adjustability of the electrodes over approximately $10^{-2}$ to $10^{-3}$ of an atomic distance: a specific length change of the piczo induces an electrode displacement, which is approximately a factor of thousand smaller. Due to the breaking process of the electrode material before a junction can be established and because the junction resistance is a controllable parameter over a large range, this device is called a mechanically controllable break (MCB) junction.

Typical dimensions of the sample geometry shown in fig. 1 are: $u \simeq 0.1-0.3 \mathrm{~mm}$ and the distance between the countersupports, $l_{s} \simeq 2 \mathrm{~cm}$. Fig. 3 shows a photograph of the central section of the sample mounting, after it was used successfully in an experiment. The adjustment of the force on the bending beam is performed by a coarse and a fine adjustment (see fig. 4). The coarse adjustment consists of spindle driven by an electromotor to move the lever part towards the bending beam. This adjustment is used to break the filament, and after this to re-establish a contact by reversing the motor direction. Once this is achieved a voltage on the piezo element is applied for fine adjustment of the constriction or the vacuum barrier between the electrodes. With this setup two different kinds of experiments are possible. In one kind of experiment the piezo voltage is kept constant, resulting in a junction with a specific resistance. In the other experiment the piezo voltage is continuously varied inducing a slowly varying resistance of the junction. This opens the possibility to monitor junction characteristics in a continuous measurement as a function of the punction resistance.

There are characteristic similarities and differences between the MCB technique and the STM technique. In the tunnel regime it is likely that in both devices tunneling occurs between two nearest atoms on opposite electrodes. In contrast to the STM, however, there is no feedback system used for the MCB junction. The two electrodes form a part of a rigid construction and can only move in one direction towards or away from each other. The possibility to scan in a lateral direction is sacrificed for a very high stability of the two electrodes with respect to each other. We estimate the absolute clectrode stability to be in the range $50-500 \mathrm{fm}$ sce, ref. [8]. Both the STM and the MCB technique make use of a piczo for the adjustment of the electrode displacement, however, the mechanisms differ substantially. Due to the geometry of the MCB sample a large retardation is established in the electrode displacement compared to the length change of the piczo, whereas in the STM the electrode displacement compared is the same as the length change of the piezo. The MCB junction is an inherently stable adjustable junction. In addition to the applications described here, this stability may prove beneficial in spectroscopic studics on single molecules. When molecules are condensated on the electrodes of a MCB junction and the electrodes are brought together, conduction is likely to take place through one or a few molecules. 

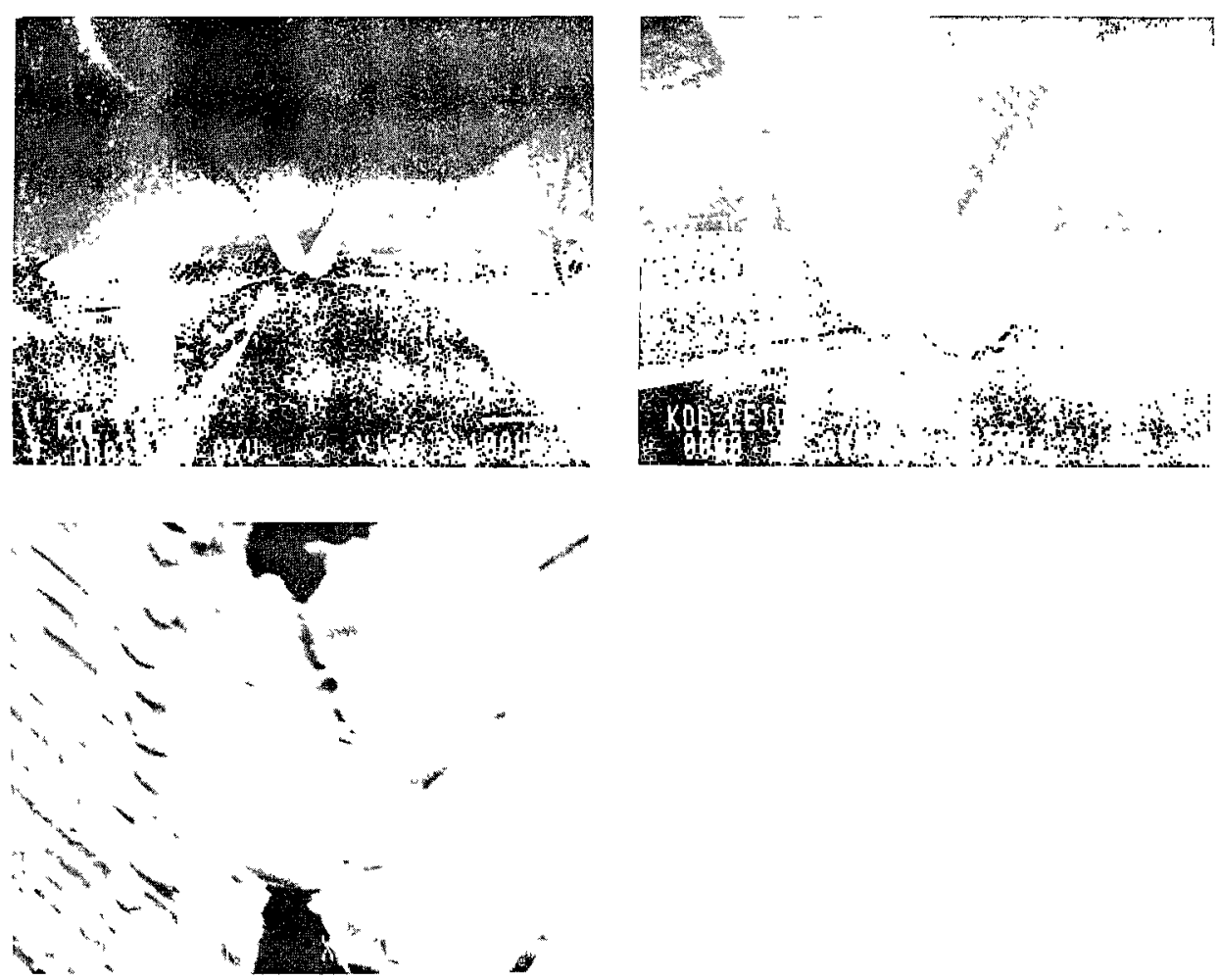

Fig. 3. An enlargement of the central part of the sample. The separated glue contacts are clearly visible in the upper leit photograph. An enlargement of the notched part of the filament is shown in the other two photographs. The photos were taken after successful operation of the sample.

In the next section measurements are presented in the contact regime, where the main attention is focussed on point contacts of atomic sizes. Unless stated otherwise the experiments have been performed in vacuum at $1.2 \mathrm{~K}$.

\section{Point contacts of platinum}

As the measurements presented here are restricted to the contact regime, the maximum resistance, which can be obtained in this regime, is the resistance for a single atom contact. For a changing piezo voltage it is easy to deduce whether the junction is in the tunnel or contact regime, because the resistance changes smoothly in the tunnel regime, whereas in the contact regime steps in the resistance occur as a function of piezo voltage. Expcrimentally we obtain a maximum resistance in the contact regime of about $10 \mathrm{k} \Omega$.

The experiment with an increasing constriction diameter is shown in fig. 5 , where two conductance traces of the same platinum sample are presented. Both traces show common features although they do not reprosicte in detail, which may be caused by some prastic deformation in the point contact region as we scan the piezo voltage, $V_{\mathrm{p}}$. For a smaller range of $V_{\mathrm{p}}$ the fine structure is reproducible as will be shown below for a niobium sample. Although we do not observe conductance quantization, discontinuous steps in the conductance occur with a magnitude of the order of $2 e^{2} / h$. When fixing the piezo voltage on a step, the point contact shows a switching behaviour between two well defined 


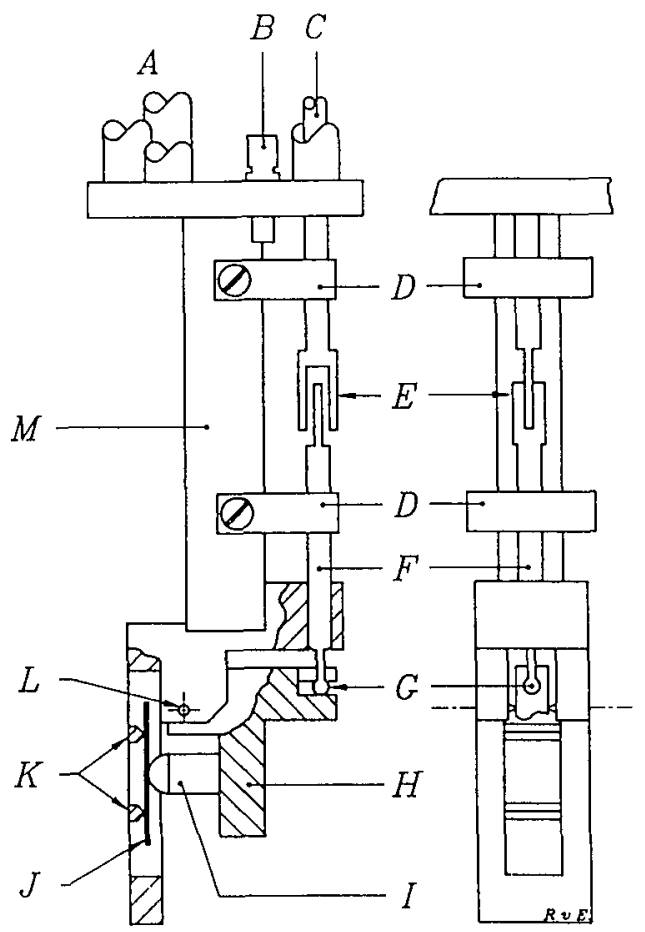

Fig 4. The mechanical adjustment part in the vacuum chamber with; A: tubes for signal and piezo lcads containing copper powder filters, B: microwave fecdthrough, C: spindle, D: bcarings, E: fork-blade coupling, $F \cdot$ screw bolt, $G$ : ballkeyhole coupling, $\mathrm{H}$ : lever, I: prezo element, J: sample mountıng, $\mathrm{K}$ : counter supports, L: turning-point, $\mathrm{M}$ : copper rod.

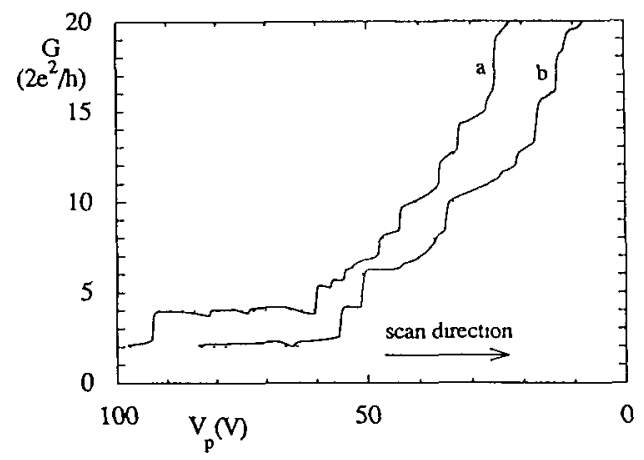

Fig. 5. Two examples of traces of the conductance, $G$, as a function of decreasing piezo voltage, i.e., increasing constriction diameter of a platinum sample. The full scan was recorded in about $20 \mathrm{~min}$. We estimate that $10 \mathrm{~V} \sim 1 \AA$. Conductance steps are of the order of $2 e^{2} / h$, but no quantization is observed. Curve a is offset in the horizontal direction with $+10 \mathrm{~V}$ for clarity. resistance states, where the switching frequency increases with the bias. Fig. 6 shows this switching effect between a 4.1 and $4.5 \mathrm{k} \Omega$ state. An $I-V$ characteristic of this switching contact, recorded in $0.1 \mathrm{~s}$, is shown in fig. 7. Similar switching behaviour between two levels, socalled two level fluctuations (TLFs), was observed by Ralls et al. [9] and Holweg et al. [10] in nano-constrictions with larger diameters compared to our atomic size point contacts.

From fig. 5 it is clear that the magnitude of the fluctuations can be as large as the conductance itself, moreover we are able to reproducibly create the TLFs and influence the duty cycle, i.e. the average time spent in one state divided by the total time. In the nano-structures of Ralls et al. and Holweg et al. TLFs are believed to be

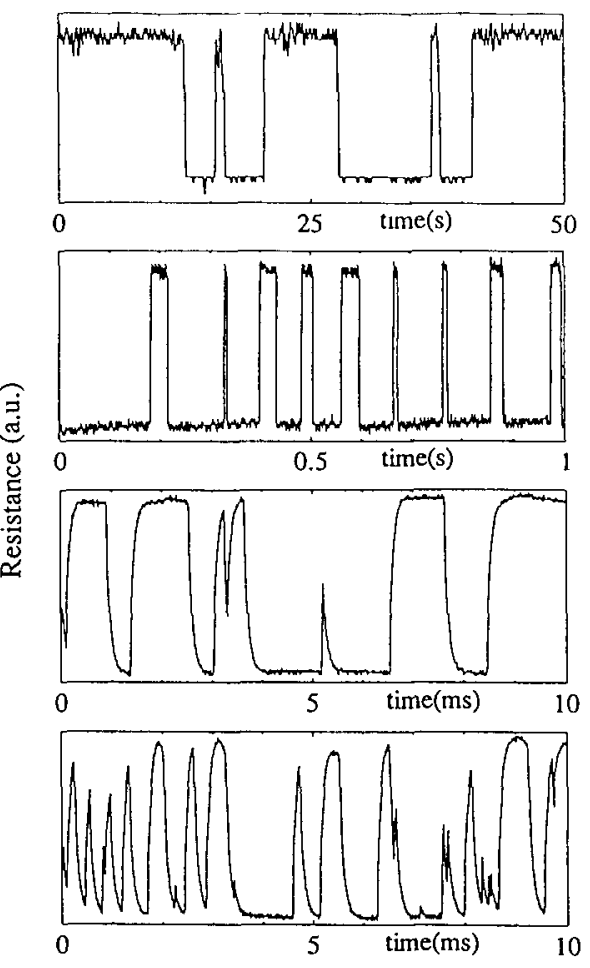

Fig. 6. Time traces at different bias currents with the piezo voltage tuned at a conductance step, which switches between 4.1 and $4.5 \mathrm{k} \Omega$. The conductance change between upper and lower level is the same in all panels, and is about $0.3\left(2 e^{2} / h\right)$. The voltages of the higher conductance state are (from top to bottom panel): $15.6,20.1,32.8,38.5 \mathrm{mV}$. The rounding of the curves in the lower panels is due to the finite time resolution of the equipment. 


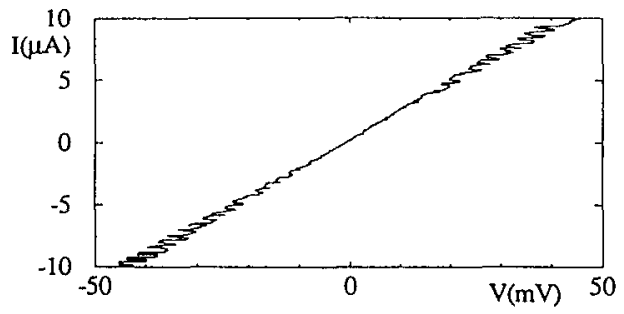

Fig. 7 An $I-V$ curve with the piezo voltage tuned at the same conductance step as in fig 6 The total recording time of this curve was $01 \mathrm{~s}$. Switching occurs at random between two different conductance valucs The switching frequency increases with increasing bias voltage, as shown in the previous figure

related to the switching of a defect between two positions in the contact. The time $\tau_{t}$ spend in a position $i(i=1,2)$ was successfully described by the expression:

$\tau_{\imath}=\tau_{0 l} \exp \left[\frac{\left(\varepsilon_{0 l}-\zeta_{l} V\right)}{k_{\mathrm{B}} T_{\mathrm{d}}}\right]$,

with $\tau_{0}$ the attempt time of state $\imath, \varepsilon_{0 l}$ the energy barrier between the two metastable positions and $\zeta_{l}$ a parameter describing current induced migration of the defect [9]. Here $T_{\mathrm{d}}$, the defect temperature, is expected to be larger than the environment temperature due to excitation by the current. Holweg et al. derived the expression $T_{\mathrm{d}}=5 e|V| / 16 k_{\mathrm{B}}$ for the defect temperature in the low temperature limit, which implies that the switching frequency increases with voltage.

In our contacts we interpret the steps in the conductance as being due to the displacement of an atom between two energetically equal positions in the constriction region. The predicted increase of switching frequency for increasing bias, eq. (1), is also observed in our contacts, as can be seen in fig. 6 . The switching times of this TLF are shown as a function of the inverse voltage in fig. 8. From this figure it appears that the attempt times $\tau_{0}$, are as high as $10^{-7} \mathrm{~s}$ and that the barrier energies $\varepsilon_{0 l} / k_{\mathrm{B}}$ are of the order of $10^{3} \mathrm{~K}$. The energy barrier is comparable, but the prefactors $\tau_{0,}$ are large compared to values obtained in refs. $[9,10]$.

An appropriate starting point for the descrip-

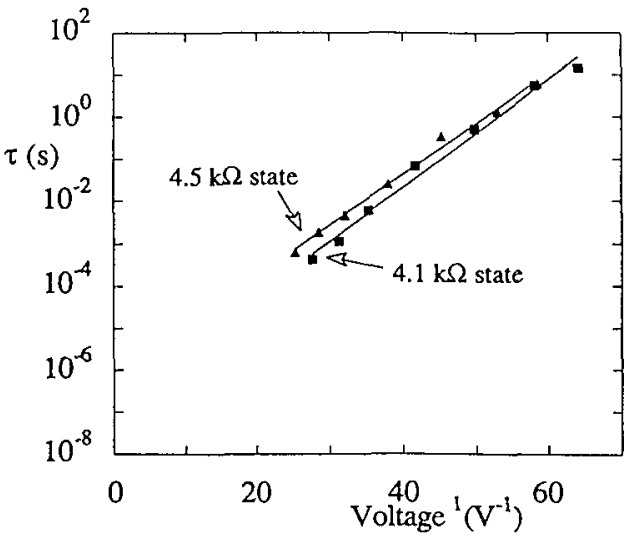

Fig 8 The average switching tımcs as a function of inverse voltage, as obtaned at different bias currents for the two level fluctuations in fig 6 The straight lines are a fit to the data From the intersection it appears that the attempt times $\tau_{0,}$ are as high as $10^{7} \mathrm{~s}$ and from the slopes it follows that the barrier energies $\varepsilon_{0,} / k_{\mathrm{B}}$ are of the order of $10^{7} \mathrm{~K}$.

tion of these contacts is the Landauer formula for the conductance $G$,

$G=\frac{2 e^{2}}{h} \sum_{n=1}^{N} T_{n}$,

where $T_{n}$ is the transmission probability of subband $n$ and $N \approx k_{\mathrm{F}}^{2} S / 4 \pi$ is the total number of occupied subbands. We have $S=\frac{1}{4} \pi d^{2}$ and $k_{\mathrm{F}} \simeq \pi / a$ so that $N \simeq(d / a)^{2} \quad(d$ and $a$ are the constriction diameter and interatomic distance respectively). Since $G$ is only a few times $2 e^{2} / h$ we conclude that our contacts are of atomic dimensions. Conductance quantization is observed when the transmission probability of the subbands is either unity or zero. Backscattering of a subband destroys the quantization. In our point contacts backscattering is likely, either because of a rapidly varying potential or due to impurities in the point contact region. The remarkable aspect of the experiment is that the displacement of a single atom can have an effect of the order of $2 e^{2} / h$ on the conductance. Since in a metallic point contact the number of subbands is of the same order as the number of atoms making up the contact, this finding is consistent with the notion of a conductance of $2 e^{2} / h$ per subband in the ballistic regime, even 
though no conductance quantization could be observed.

\section{Point contacts of superconducting niobium}

Superconducting electrodes are interesting because apart from the resistance an additional parameter, being the critical current, can be studied. The product of the critical current $I_{c}$, and the normal resistance, $R_{\mathrm{N}}$, of a point contact represents a characteristic voltage of the order of $\Delta / e$. For a short weak link (as compared to the coherence length) Kulik and Omelyanchuk showed that, in the classical limit of constriction width much greater than Fermi wave length, the $I_{\mathrm{c}} R_{\mathrm{N}}$ product is constant, independent of $R_{\mathrm{N}}$. They analyzed two possible limits, a dirty contact where the electron mean free path is short compared to the constriction diameter [11] (KO1) and a clean, ballistic, contact where the electron mean free path is much larger than the constriction diameter [12] (KO-2). In the zero temperature limit the calculated $I_{\mathrm{c}} R_{\mathrm{N}}$ value for a clean contact is $\pi \Delta / e$. In a recent paper [4] it was shown that this relation survives under conditions that $R_{\mathrm{N}}$ is quantized. As a result, $I_{c}$ was predicted to increase stepwise, as a function of the constriction diameter, with steps equal to $e \Delta / \hbar$.

We have measured a large number of $I-V$ curves at $4.2 \mathrm{~K}$, where the resistance is varied over more than three orders of magnitude, see fig. 9. The normal resistance is derived from a linear fit to the part of the curve between \pm 5 and $\pm 10 \mathrm{mV}$. For every point contact the $I_{\mathrm{c}} R_{\mathrm{N}}$ product is thus obtained. These data points, together with the data of another MCB sample mcasured at $1.2 \mathrm{~K}$, are given in fig. 10 as a function of the normal resistance. Also indicated in this figure are the zero temperature predictions of Kulik and Omelyanchuk, KO-1 and KO-2 [11,12].

The interpretation of this diagram is not yet clear to us. In the low resistance part of the diagram, below $80 \Omega$, it is suspected that heating effects [13] or non-equilibrium effects [14] have their influence on the value of the $I_{\mathrm{c}} R_{\mathrm{N}}$ product. An interesting part of fig. 10 is the resistance
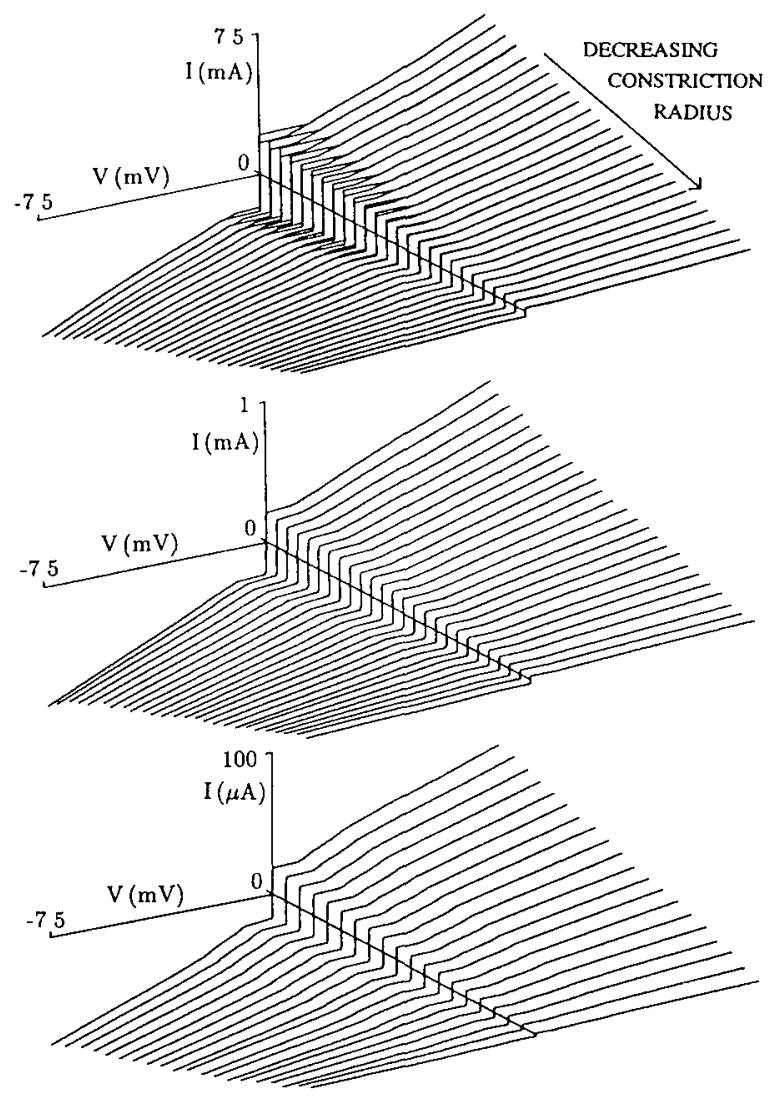

Fig $9 I-V$ chadacteristics of a nobium sample at $42 \mathrm{~K}$ The normal resistance is increased by diminishing the constriction radius, resulting in a resistance change from approximately $1 \Omega$ to $1 \mathrm{k} \Omega$

range exceeding $80 \Omega$. In this range a uniform logarithmic decrease of the $I_{\mathrm{c}} R_{\mathrm{N}}$ product is observed for two different samples at two different temperatures. One might think that for large normal resistances the $I_{\mathrm{c}} R_{\mathrm{N}}$ product should be described by the theoretical AmbegaokarBaratoff value [15], $\pi \Delta / 2 e$, for a tunnel junction ( $2.2 \mathrm{mV}$ for niobium). However, we stress that in the contacts below $10 \mathrm{k} \Omega$ no tunneling occurs, and therefore these contacts should be described by one of the two theoretical models of Kulik and Omelyanchuk. We argue that the $I_{c} R_{\mathrm{N}}$ decrease may find its origin in a reduction of the damping of the system. The amount of damping in a Josephsons junction is described by the McCumber parameter [16], $\beta_{\mathrm{c}}$. Although our point contacts have a low capacitance, due to 


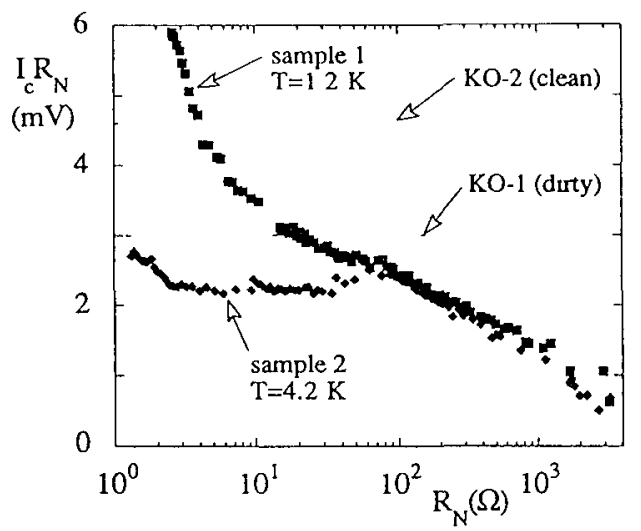

Fig 10) The $I_{c} R_{\mathrm{N}}$ products as a function of $R_{\mathrm{N}}$ on a logarithmic scale for two samples at two difterent temperdtures The predictions for the short dirty, $\mathrm{KO}-1$, and the short ciean, $\mathrm{KO}-2$, weak link are indicated At the low impedance side there is a steep increase in $I_{\mathrm{c}} R_{\mathrm{N}}$ for decreasing $R_{\mathrm{N}}$ At $R_{\mathrm{N}}$ values exceeding $80 \Omega$ a uniform decrease of $I_{\mathrm{c}} R_{\mathrm{N}}$ for increasing $R_{\mathrm{N}}$ is observed

their high normal resistance they are in the underdamped regime, $\beta_{c} \gg 1$. When a fluctuation mechanism (thermal, electrical, quantum) induces a system to switch to the voltage carrying state before it has reached its maximum theoretical critical current, the low damping will make it easy for the system to remain there. Therefore, a reduced damping together with a fluctuation mechanısm can explain the observed reduction of $I_{\mathrm{c}} R_{\mathrm{N}}$ for increasing $R_{\mathrm{N}}$. However, that this decrease of the $I_{\mathrm{c}} R_{\mathrm{N}}$ product at $R_{\mathrm{N}}$ values larger than $80 \Omega$ has a logarithmic dependence remains to be explained.

We now discuss the measurement of $R_{\mathrm{N}}$ and $I_{\mathrm{c}}$ of atomic size niobium point contacts as a function of continuously varying piezo voltage. The critical current is measured at a setpoint voltage near $V=0$, such that the critucal current and the setpoint current coincide within $0.1 \%$. The current biasing the sample is ramped until the setpoint voltage is reached. Then the setpoint current value is held at an output terminal while the current bias switches back to zero. This procedure is repeated periodically, which enables us to measure the critical current value while the constriction diameter is varied. For two periods of the piezo voltage such a measurement

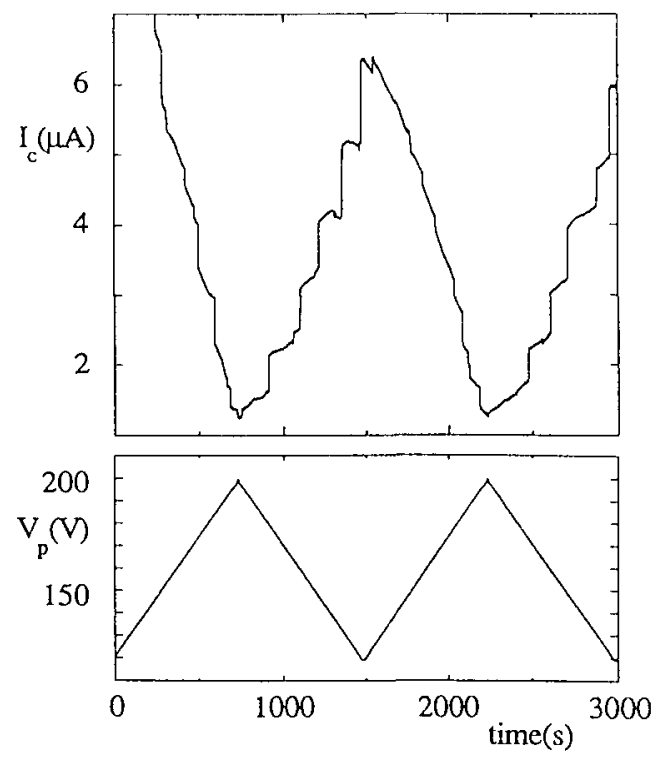

Fig 11 A critical cuirent medsurement of a nobitum sample over two periods of the triangular piezo voltage For an increasing constriction diameter (decreasing piezo voltage) steps in critical current of the order of $e \Delta / \hbar \approx 04 \mu \mathrm{A}$. are observed The difference in the curves for increasing and decreasing $V_{p}$ may reficct d difference in stress in the point contact region

is presented in fig. 11. Clear critical current steps occur when the constriction diameter changes. The step size is of the order of $e \Delta / \hbar \simeq 0.4 \mu \mathrm{A}$, consistent with the theoretical prediction of a critical current $e \Delta / \hbar$ per subband in a superconducting quantum point contact [4]. The slopes for decreasing $V_{\mathrm{p}}$ are different from those for increasing $V_{\mathrm{p}}$, which may be due to a different stress in the point contact region when the electrodes are moved apart compared to the situation where the electrodes are pressed into each other. Although the rough shape of the two slopes for increasing and decreasing $V_{\mathrm{p}}$ are similar, the detailed structure does not reproduce. As in the case of the platinum contact we expect that in the used $V_{\mathrm{p}}$ amplitude of $80 \mathrm{~V}$ some plastic deformation in the point contact region occurs, so that the corresponding slopes do not reproduce in detail.

In fig. 12 we show an analogous measurement as in fig. 11 over three periods of $V_{p}$ with a smaller $V_{\mathrm{p}}$ amplitude $(25 \mathrm{~V})$. In this graph a 

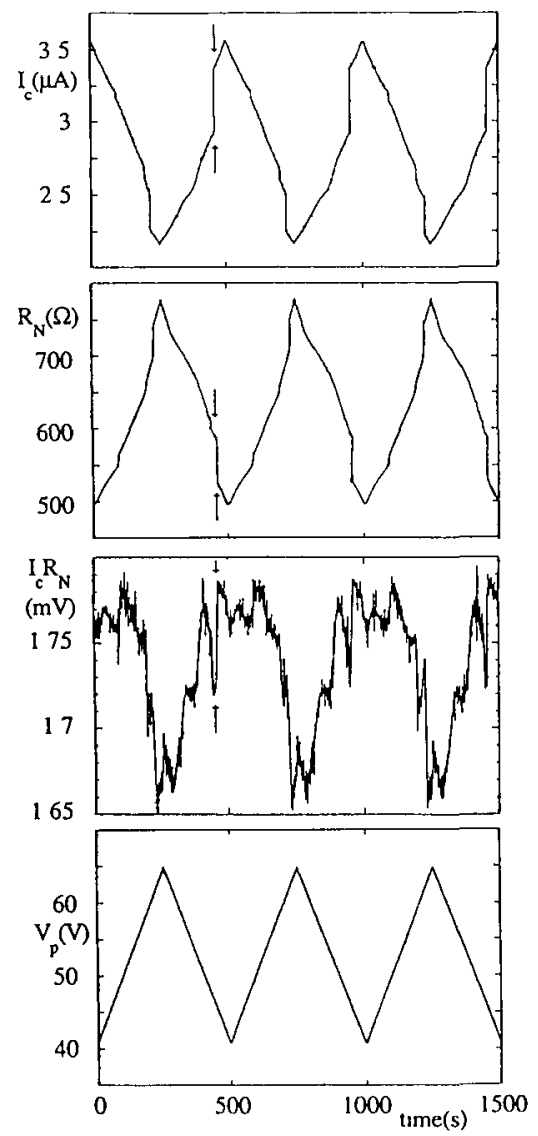

Fig 12 Critical current and normal resistance measurement on a smaller piezo voltage range as compared to fig 11 The fine structure in $I_{\mathrm{c}}$ lines up with that in $R_{\mathrm{N}}$ and $I_{\mathrm{c}} R_{\mathrm{N}}$ The relatively large changes in $I_{c}$ and $R_{\mathrm{N}}$ at the steps almost compensate each other in the product $I_{\mathrm{c}} R_{\mathrm{N}}$

resistance measurement is included, measured by a lock-in amplifier at voltages larger than $2 \Delta / e$. This measurement is performed after the $I_{\mathrm{c}}$ measurement with the same $V_{\mathrm{p}}$ scan, where the minimum in $V_{\mathrm{p}}$ served as a zero time reference. The third panel shows the product of the $I_{\mathrm{c}}$ and the $R_{\mathrm{N}}$ values. It is clear from the top three panels that this measurement reproduces perfectly, even the fine structure of the $I_{\mathrm{c}} R_{\mathrm{N}}$ product reproduces with the period in $V_{\mathrm{p}}$. This figure shows that it is possible to move one or a few atoms in a reproducible way in and out of a constriction. The discontinuities in $I_{c}$ line up perfectly with the discontinuities in $R_{\mathrm{N}}$ and $I_{\mathrm{c}} R_{\mathrm{N}}$, as can also be seen from fig. 13, which shows the

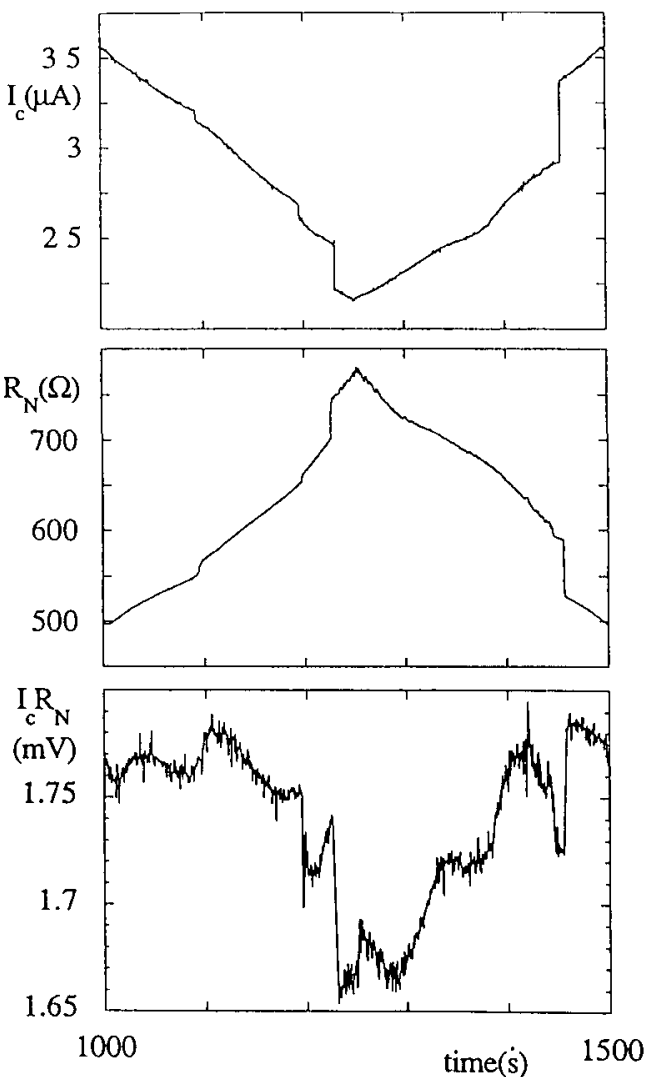

Fig 13 One period of fig 12 on an expanded scale

last period of fig. 12 on an expanded scale. The variations of approximately $50 \%$ in $I_{\mathrm{c}}$ and $R_{\mathrm{N}}$ separately almost compensate and are reduced to about $7 \%$ variations in the product. The values of the $I_{\mathrm{c}} R_{\mathrm{N}}$ product for the $R_{\mathrm{N}}$ values considered $(500-750 \Omega)$ with an average of $1.7 \mathrm{mV}$, are in agreement with the values presented for two different samples in fig. 10 .

\section{Conclusions}

In summary, the present technique allows measurements of point contacts and tunnel junctions, of which the resistance can be precisely adjusted, even in the regime of a contact consisting of only a few atoms. The change of conductance of a point contact when the electrodes are pressed into each other is determined mainly by the atomic structure of the contact, resulting in 
well resolved steps in the conductance Application of the measurement technique to metals with a large Fermi wave length compared to the atomic dimensions (semimetals) may allow the observation of conductance quantization We have presented measurements on adjustable point contacts of normal and superconductıng metals With the present technique it is possible to measure junction characteristics, such as $I_{c}$ and $I_{\mathrm{c}} R_{\mathrm{N}}$ with the junction resistance as a parameter When the constriction diameter is changed a stepwise variation in the critical current is observed, coinciding with steps in the conductance The steps in $G$ and $I_{c}$ are of the order of $2 e^{2} / h$ and $e \Delta / \hbar$, respectively

\section{Acknowledgement}

We wish to thank prof $\mathrm{L} \mathbf{J}$ de Jongh for his interest and for stimulatıng discussions

\section{References}

[1] B J van Wees, H van Houten, C W J Beenakker, J G Willamson, L P Kouwenhoven, D van der Marel and C T Foxon Phys Rev Lett 60 (1988) 848,

D A Wharam T J Thornton R Newbury, M Pepper, H Ahmed, J E F Frost, D G Hasko, D C Peacock, D A Ritchie and G A C Jones, J Phys C 21 (1988) L209

[2] E A Montie E C Cosman, GW t Hooft, M B van der Mark and CW J Beenakker, Nature 350 (1991) 594

[3] CW J Beenakker and $\mathrm{H}$ van Houten, Solıd State Phys 44 (1991) 1
[4] CW J Beenakker and $H$ van Houten Phys Rev Lett 66 (1991) 3056,

C W J Beenakker Phys Rev Lett 67 (1991) 3836

[5] C J Muller, J M van Ruitenbeek and L J de Jongh, Physicd C 191 (1992) 485

[6] C J Muller, J M van Ruitenbeek and L J de Jongh, Phys Rev Lett 69 (1992) 140

[7] J Moreland and J W Ekin, J Appl Phys 58 (1985) 3888

[8] Note that the atomic zero point vibration amplitudes are much larger than the stability quoted here The atomic vibration frequencies are of the order of phonon fre quencies $\approx 10^{17} \mathrm{~Hz}$ and the amplitudes are estımated to be several picometers The experimental frequency window however, has its upper limit at about $10^{4} \mathrm{~Hz}$, implying that in our experiment the atomic positions are averaged over the vibration amplitudes, which is only $\approx 1 \%$ of the atomic size The referred stability is determined by the observed random variations of the resistance, $1 \mathrm{e}$ electrode spacing, and therefore only sensitive to frequencies within the experimental window

[9] K S Ralls, D C Ralph and R A Buhrman, Phys Rev B 40 (1989) 11561

[10] PA M Holweg, J Caro, A H Verbruggen and $\mathrm{S}$ Radelaar, Phys Rev $B$ to be published

[11] I O Kullk and A N Omelyanchuk, Pıs'ma Zh Eksp Teor F1z 21 (1975) 216 [JETP Lett 21 (1975) 96]

[12] I O Kulık and A N Omelyanchuk, Fiz Nizk Temp 3 (1977) 945 [Sov J Low Temp Phys 3 (1977) 459]

[13] VN Gubankov, K K Likharev and N M Margolın, Fiz Tverd Tela 14 (1972) 953 [Sov Phys -Solid State 14 (1972) 819],

T A Fulton and L N Dunkleberger, J Appl Phys 45 (1974) 2283

[14] I K Yanson, personal communication

[15] V Ambegaokar and A Baratoff, Phys Rev Lett 10 (1963) 486, Phys Rev Lett 11 (1963) 104

[16] D E McCumber, J Appl Phys 39 (1968) 3113, W C Stewart, Appl Phys Lett 12 (1968) 277 